Association for Information Systems AIS Electronic Library (AISeL)

International Research Workshop on IT Project International Research Workshop on IT Project

Management 2009

Management (IRWITPM)

12-14-2009

\title{
Using Realist Social Theory to Explain Project Outcomes
}

Michael J. Cuellar

North Carolina Central University, mcuellar@georgiasouthern.edu

Follow this and additional works at: http://aisel.aisnet.org/irwitpm2009

\section{Recommended Citation}

Cuellar, Michael J., "Using Realist Social Theory to Explain Project Outcomes" (2009). International Research Workshop on IT Project Management 2009.7.

http://aisel.aisnet.org/irwitpm2009/7

This material is brought to you by the International Research Workshop on IT Project Management (IRWITPM) at AIS Electronic Library (AISeL). It has been accepted for inclusion in International Research Workshop on IT Project Management 2009 by an authorized administrator of AIS Electronic Library (AISeL). For more information, please contact elibrary@aisnet.org. 


\title{
Using Realist Social Theory to Explain Project Outcomes
}

\author{
Michael J. Cuellar \\ North Carolina Central University \\ mcuellar@nccu.edu
}

ABSTRACT

In researching IS phenomena, many different theoretical lenses have been advanced. This paper proposes the use of Margaret Archer's Morphogenetic Approach to Analytical Dualism (MAAD) as a social theoretic approach to explain why social phenomena may occur in a case study. This paper provides a brief overview to MAAD, providing a brief description of its tenets and methodology for use in an empirical study. Then as an example, it applies MAAD to the well-known CONFIG case (Keil, 1995). This approach shows that the implementation failed due to the forces of the ideological structures on the project team. Further, it finds that the project escalated due to the prevailing corporate culture and credibility of the advocate. The project was de-escalated when the culture was changed and the advocate died. This example shows that the use of this social theory can provide explanatory purchase where social phenomena are involved.

\section{Keywords}

Social Theory, Critical Realism, Morphogenetic Approach, Analytical Dualism, Margaret Archer, Case Study, Research Methods

\section{INTRODUCTION}

Project management has been a problematic area of practice in the field of information systems. Despite progress made in methods and much research the field, many projects still fail, escalate or otherwise fail to achieve their goals (Rubenstein 2007). The search for explanations for these failures has shown that there are many reasons. For example, just in the area of project escalation alone, many reasons have been advanced which fit into many categories: project, psychological, social, contextual and organizational. Additionally, escalation has been seen as a phenomenon that has more than one, often many causes in a particular instance of escalation (Staw, 1997). Thus in looking at case studies of project related phenomena, it seems logical to seek to find a methodological approach that will drive out these causal mechanisms and bring them to light.

In particular, it would be desired to be able to identify why a project was or was not completed or why the implementation in an organization was successful or not successful. Both of these may be thought of as instances of change. In the first, why a project failed to complete a useable artifact is a case of change in the sense of creating a new artifact. In the second, it is a case of change of organizational routines. Methodologically, we need an approach that allows us to identify the causal mechanisms as to why the desired change failed to occur.

Many different approaches can be found for analyzing case data: grounded theory, phenomenology, even hypothesis testing have been advocated (Lee, 1989) as well as less formal approaches (Eisenhardt, 1989). While these approaches all have the merits, none of them provides a mechanism to specifically focus on why change occurred or didn’t occur.

In examining why change occurred or did not occur, a key aspect of IS project work that must be considered is that it is a social phenomenon. It is not an individual activity but one in which groups of people interact either in harmony or in conflict. In developing an information system groups of developers and users interact to bring the IT artifact into existence. Similarly, when an artifact is implemented into an organization, the developers and users interact to create a new business system that incorporates the artifact. The resultant artifact or implementation of an artifact is rarely that which is intended by any one of the parties. Rather, it is usually the outcome of the negotiated interactions between the parties. Social theory, the use of theoretical frameworks to study and interpret social structures and phenomena (Wikipedia 2009) is one way that we can analyze this type of interaction to explain how change occurs in social situations.

Structuration theory (ST) (Jones, Orlikowski et al. 2004; Jones and Karsten 2008) has been applied in a wide variety of situations in the IS literature (Jones and Karsten 2008). While it has been widely utilized in the IS literature, it has been subject to a number of criticisms. For example, in general sociology it has been criticized for an a-historicity in its model of relations between structure and agency (Archer 1995). Because of its melding of structure and agency, analysis can only take place in the "now". Examination of the effects of previous structures is ruled out since they were constituted by different people than those here present. Another issue is the over-privileging of agency with respect to structure. Since social 
structures are regularized social practices, they don't exist until instantiated by actors. Thus they have no relatively enduring existence apart from the actors. Therefore, while they actors draw upon them to inform their practice, they cannot have any causal influence over the actors.

The handling of technology within ST has also been problematic. Rose, Jones and Truex (2005) claim that ST unduly privileges human agency which causes technology to vanish into being simply "an occasion for structuring". As dealt with in Orlikowski (2000), information technology is held to be a non-actor. It is treated as a multi-faceted tool from which users select features to be employed in structuring the organizations. In Orlikowski (2005), she seems to recognize this and advocates that we need to look at "different conceptual treatments of human and technological agencies" (p. 185). She recognizes a different between human and technological agency preferring to call the later "technological performativity" of settings.

It therefore seems to make sense to look at another form of social theory that might avoid these criticisms and might be able to assist us in examining project outcomes. One such theory is Archer's Morphogenetic Approach to Analytical Dualism (MAAD) (Archer 1988; Archer 1995). This theory as shall be seen below resolves many of the issues associated with ST. As opposed to the a-historicity of ST, MAAD specifically includes time into the analysis in the form of the Morphogenetic Cycle that will be explained below. Similarly, structure and agency are held apart analytical in a dualism rather than the conflationary duality used by ST. Thus neither structure nor agency is privileged. Finally, IT artifacts can find a place as a material structure within the framework of MAAD thus allowing it to be introduced into the analysis.

This paper therefore seeks to describe (MAAD and to demonstrate how it can be used to explain project outcomes by means of a secondary case study. MAAD is a general social theory developed specifically to assist in identifying why change in social structures occurred or did not occur and thus seems to be ideal for use in analyzing project outcomes. The rest of the paper describes Archer's theory and then show how it applies to analysis of a case of project escalation.

\section{A DESCRIPTION OF MAAD}

This section describes the basic tenets of Archer's Morphogenetic Approach to Analytical Dualism (AD). It describes the basic components of AD: structures, agencies and the morphogenetic cycle.

\section{Components: Material and Ideational Structures}

Social structures are considered to be emergent properties of relationships between social entities that as such are irreducible to those entities, relatively autonomous in their influence and relatively enduring. They are considered emergent in that they arise from interactions between agencies. Structures have properties that allow them influence the world around them. Structures pre-date any particular set of individuals and thus can be only reproduced or transformed by social action (Archer 1995, p. 168).

Structures are considered to be of two types: material structures and ideational structures (or culture). Material structures consist of relationships between individuals, and other material structures. In material structures, the relationships are between role-positions rather than incumbents of those positions. In a marriage relationship, for example, husband and wife are role positions which exist independently of who the husband and wife are. Thus the existing material structure pre-exists any social interaction based on or conditioned by it and every change or elaboration of the structure post-dates the interaction. Thus every couple marrying reproduces or transforms the existing marriage structure. Any transformation of the structure post-dates the interaction of the agents.

Ideational structures (also called cultures or cultural systems) are logical relationships between propositions. Cultural systems are extracted from the sum total of existing "intelligibilia” which roughly corresponds with Popper's World Three (Archer 1988). These propositions stand in objective logical relationship with each other. For example, some propositions might be A: "all men are created equal", B; "in Christ, all men are equal", C: "black men are not socially equal to white men" and D: "blacks should be subservient to whites." If these four propositions are part of the cultural system, propositions A and B are complementary to each other as are C and D. However propositions A and B are contradictory to C and D. As logical relationships, the cultural system is independent of any society or cultural group that holds it. Should no group espouse it, it still can exist in the accumulated cultural library of the society where it can be extracted and advocated for.

\section{Components: Agency}


Agencies are groups of social actors going through the same life experiences. These social actors in turn are derived from Persons who assume roles within the Agency. Persons are viewed as reflexive agents. They can examine their current existence and circumstances and decide to continue or discontinue their existing situation. Actors derive from a relationship between the people and the agency. A person is born and socialized within a particular agency. As s/he comes to maturity, $\mathrm{s} /$ he can choose to take a role as an actor within that agency or seek to move to a different agency. Actors are thus a particular social identity taken on by a person as their role within an agency.

There are two kinds of agencies: primary and corporate. Primary agents are simply collectivities of individuals sharing a life experience. They are not organized and have neither leadership nor a message to articulate. A corporate agent is a group of persons who have organized to take action. They have a set of roles and leadership which articulates a message and seeks to execute an action as a group.

These agencies have different distributions of resources (wealth, sanction or expertise). This distribution either enables or disables the agencies for undertaking desired projects.

\section{Components: Morphogenetic Cycle}

The link between the structures and the agencies in terms of defining how structural change occurs is the morphogenetic cycle (figure 1). Previous cycles have created a particular set of existing structures and distributions of resources as the result of prior cycles which condition the actions of existing agencies creating opportunity costs to intended actions which

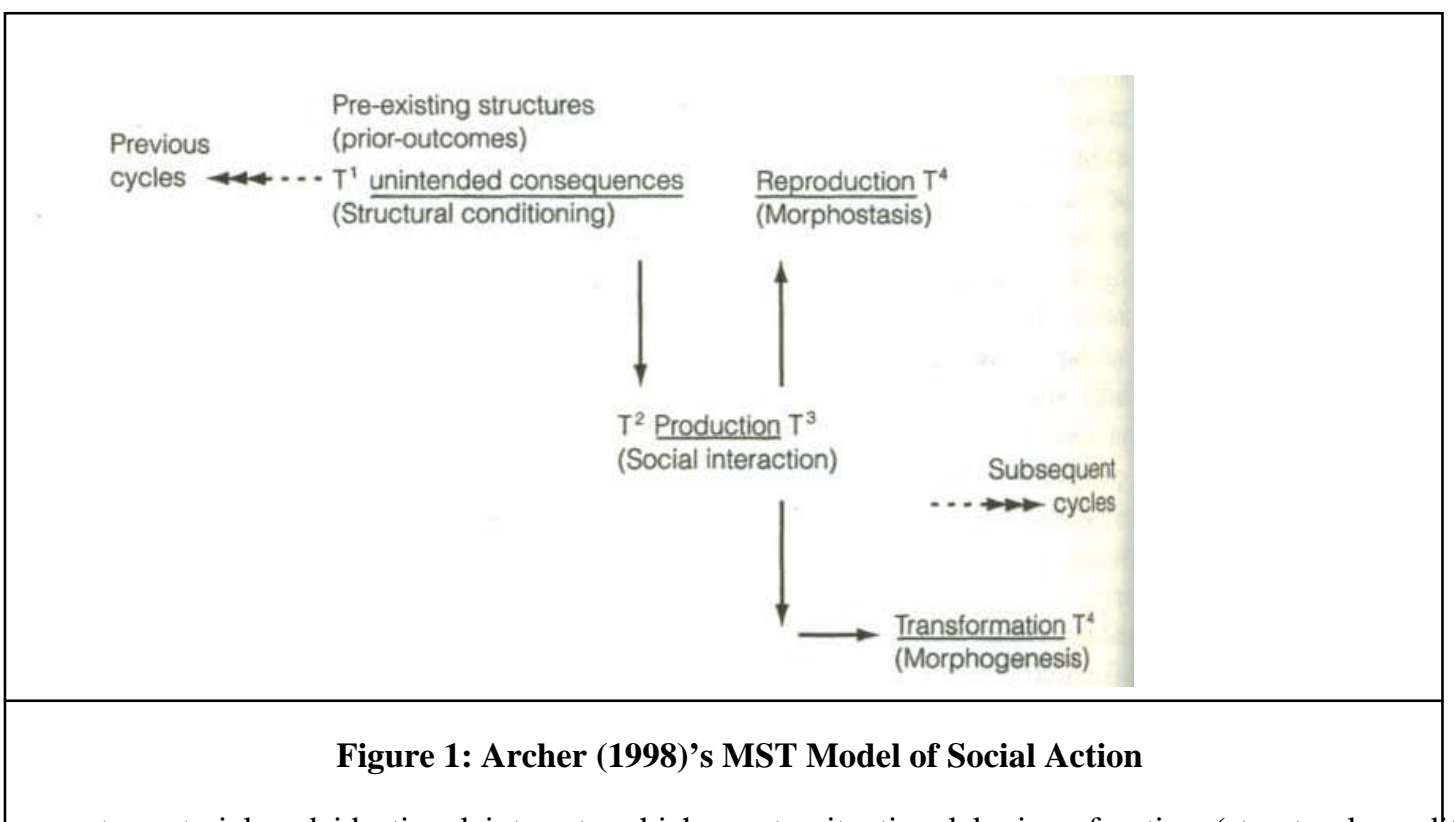

in turn credte material and ideationat interests whichr create situational logics of action (structurat conchtioning). As the agencies act to achieve their interests (Social Interaction), agencies and resources are redistributed which in turn changes the balance of power leading to the reproduction or transformation of structures (Morphostasis/Morphogenesis).

This cycle is an analytical distinction rather than an ontological one. Action is constantly going on and there is no static point. However, it is possible to abstract from reality salient features of societal change actions that are desired to be analyzed. These situations can be analyzed by means of this device. We will now look at each phase in more detail.

\section{Structural Conditioning}

Phase I of the morphogenetic cycle, Structural Conditioning is the result of the actions of previous agents. The "first order effects" of these actions are the conditions in which the agents find themselves. These conditions have three effects on agents: 1) they enable certain actions and discourage or penalize others; 2) they create certain opportunity costs for pursuing certain actions; 3 ) they also allocate various distributions of resources among the agents. These various conditions create a vested interest in the agent of either preserving or changing the status quo. 
Conditioning also provides "direction guidance” (Archer 1995, p. 213). The properties of the structures and cultures that exist interact to provide this guidance. These properties can be required by the nature of their relationship (e.g. employeremployee, manager-team member, husband-wife) in that they cannot exist without the other. Or their relationships can be contingent, only incidentally related (e.g. all people of a certain age). Additionally, the relationship between the properties can be one of coherence or contradiction. Archer calls these "second order effects" (Archer 1995, p. 215) as they arise from the first order effects described above.

These interactions give rise to "situational logics" (Archer 1995, pp. 216-218). Where the relationships between elements of a structure are necessary and where they are compatible with each other, agents are motivated by their vested interests and opportunity costs of change to protect the existing structures. Similarly, where relationships are necessary but are incompatible with each other, those in power are motivated to compromise in order to retain their structure. Where relationships are not essential to the structure and are incompatible, it is possible to seek to directly eliminate the incompatibility either to preserve the status quo or to create revolutionary change. Lastly, where the structures are not required but are compatible, it fosters an attitude of opportunism: to create new potentials by exploiting new capabilities to increase the capabilities of the agents.

\section{Social Interaction}

Phase 2 of the morphogenetic cycle, social interaction, begins when one or more agencies decide to make an effort to effect change according to their situational logics, creating environmental pressures. This shapes the context for all actors. Other agencies react to these pressures. As the agencies interact, the various agents constrain and enable each other and the various agencies are redefined over time. The net result is that at the end of the process, there is realignment of the relationships between the agents with regard to size, constituency, and resource allocation. The original resource (management sanction, wealth and expertise) distribution that existed at the time of structural condition is now redistributed among the realigned agencies.

\section{Transformation}

In this final phase, transformation or social elaboration, the effects of the social interaction in the previous phase are worked out. This is accomplished by transactions between the realigned agents carried out by means of exchange and power. This works out by engaging one of three different types of interaction: confluence of desires, where all parties are agreed on the course of action; reciprocal exchange, where each gives something in exchange for a gain in another area; and power-induced compliance, where the will of one agent is forced on the others. The agents' ability to enact their desired situation logic is based on their access to resources. This interaction of the agents with their resource levels creates their bargaining power which in turn determines their ability to carry out the actions that they desire per their situational logic.

Bargaining power however is a necessary but not sufficient condition for the definition of an exchange because a transaction requires us to consider the bargaining power of each participant in the exchange to determine a successful transaction. This leads to the concept of negotiating strength. Depending on the resources possessed and needed by each partner, the negotiating power of an agent will vary. Where one agent is dependent on another for certain resources, the transaction will tend to be transacted in terms of power while where the resource requirements are evenly distributed, it will tend to be more of an exchange.

This negotiating strength then is exerted by Corporate Agents who seek to enact their desired social state in methods derived from their situational logic. Where negotiating strength is relatively equal, the new social arrangements are negotiated. Where there is a disparity in strength, the new social arrangements can be imposed by power. Whichever is the case, the resulting social/cultural structure is not predictable.

In the next section, the paper describes a methodology for how to apply this approach in IS research and then uses it in an example secondary case study.

\section{A METHODOLOGY FOR USING ARCHER'S SOCIAL THEORY IN IS RESEARCH}

Archer describes a basic process for doing explanatory research (Archer 1995). This explanation takes the form of "analytic histories". In these histories, we describe the morphogenetic cycle that occurred for the social phenomenon under study. We describe the structural conditions that existed and how these impact the agencies in play during the sequence. The agencies, structures, vested interests, situational logics and resource distributions should be examined. Next, we describe the interaction 
that occurred an how the agencies changed and realigned during the cycle. Changes in resource distributions should be examined. Finally, we discuss the changed bargaining power of the agencies, which led to changes in negotiating strength and power relationships. Then we should describe the nature of the negotiations over the form of the structures that took place and why the resulting structures took the form that they did.

\section{EXAMPLE CASE STUDY}

To demonstrate the capability of this methodology, this section reviews the "CONFIG" case study (Keil, 1995), which is a study in project escalation. This case was chosen as it is a well-known secondary case and thus is readily accessible to the readers. Additionally, it contains sufficient detail to enable the morphogenetic analysis process.

In this case, CompuSys, a large computer company, which manufactured highly configured computer systems, had identified a problem in system configuration: orders were being misconfigured in the sales process which resulted in many allowances to the customer and provision of many parts at no cost to the customer. This problem was costing the company millions of dollars. To resolve this problem, Tom Jones and a team of developers, sponsored by the manufacturing organization, built VERIFIER, an expert system that checked configurations of the systems prior to shipment to the customer. VERIFIER was a highly successful effort resulting in saving of millions of dollars to the customer. As a result, Jones gained high status and respect within the organization. This success also led Jones and his team to seek to expand the VERIFIER technology to other areas. This set the stage for the morphogenetic cycle leading to the development and implementation of the CONFIG system. To illustrate the MAAD approach to analyzing change situations, this study examines the initial development of the system to deployment in detail and analyzes the escalation and de-escalation of the project in summary fashion.

\section{Structural Conditions at the Start of the Cycle}

Jones and his team had determined on the project of changing the sales process to include the use of an automated configuration checking process. As we look at this situation, we can identify two agencies: 1) Tom Jones and Team, which is s a corporate agency. They were organized and had a message to take to the organization; and 2) the Sales organization which was a primary agency as it had no organized opposition to the implementation of the system.

The ideological structures involved in this situation contained the following propositions

1. 1. The performance of the organization should be improved over time

2. 2. Organizations that run their own business and don't coordinate between themselves are the best way to organize the company (Stove Pipe Mentality)

3. 3. We should respect Tom Jones for his ability to resolve configuration problems and improve organizational performance

4. 4. Configuration errors begin in Sales

5. 5. Configuration errors cost money and should be corrected,

6. 6. The errors introduced by poor configurations created in the sales organization were generally small and not necessary to be resolved in the sales organization

7. 7. Compensation of the Sales People is based on sales not on configuration accuracy

8. 8. There was a culture around R\&D type projects such as this that allowed these projects to continue. There was a philosophy that "we don't kill projects", "we support strong advocates of projects, " and there was an entrepreneurial money raising culture that allowed pet projects such as CONFIG to continue

9.

The manufacturing organization, led by Tom Jones, was interested in resolving a contradiction between propositions 1 and 6. They felt the results of these misconfigurations were substantial and wished to have these issues resolved. They wished to substitute the idea that sales people should make the effort to configure their sales correctly. This represents a competitive contradiction where the manufacturing organization offered a competitive idea to the existing ideology. This represents a situational logic of elimination.

The material structures involved are the sales process and the sales compensation system. In the sales process, there is a necessary contradiction involved in it. In order for the sales to be fulfilled it was necessary that sales send orders to the manufacturing group, however because they did not check for configuration accuracy, the manufacturing group was faced with correcting problems that occurred. The structure, the sales process, was necessary because one part could not dispense with the other but the incompatibility of the output from the sales process to the manufacturing process represents cleavage in the process. 
By virtue of the existing sales compensation system, the salespeople had no incentive to adopt the additional work of checking the configurations of the quotes that they created. In fact, the record shows that they were under considerable pressure to respond quickly to requests for quotes and felt that they could not take the time to use a time-consuming quote verification system. For the sales people, it was in their interest to shorten the cycle for quotations. And since their compensation was tied to sales only not the correctness of the configuration of their quotes, it was not in their interest to add anything that got in the way of their making a sale. They thus had a vested interest in maintaining the sales process as it was. For them, while there was a contradiction between the recognition that quotations were being incorrectly configured which cost the company money and the desire to improve the performance of the company, they covered that over with thought that they were paid to sell, not configure, and that the configuration errors were not about big items only small ones. Theirs was the opinion that this contradiction was necessary to the maintenance of their vested interest. Thus they had a situational logic of preservation.

Jones' team was motivated by the desire to change the sales process to eliminate the configuration errors. While VERIFIER was successful in saving millions of dollars by resolving configuration errors before they reached the customer, it did not resolve the source of the problem, which was poor configuration in the sales cycle. As part of the manufacturing organization, they had a vested interest in removing this problem. There was a contradiction in the structure between the goal of producing cost effective implementations of product and the input from the sales cycle. The sales cycle involved necessary relationships between the organizations, therefore Jones and team adopted a situational a situational logic of correction.

In terms of resources, Jones and his team had the expertise to build the CONFIG system, got the material resources that they would need for it and had the sanction to build it from the manufacturing organization. They also had sanction in the form of Jones' credibility as someone who has significantly benefitted the organization. However, what they lacked was the sanction to implement the system. The sales team had the sanction, by virtue of the stovepipe organizational form, to control the structure of the sales process. A stovepipe organizational form is used here to indicate that the sales and manufacturing organizations operated as separate "fiefdoms" which determined their own success criteria without regard to the other. Thus each organization had only a small amount of influence on the other. Jones would have to work via negotiation to implement the system into the sales process. The sales organization possessed the sanction via the stovepipe organizational structure in place to control any change that occurred within their organization.

\section{Social Interaction}

The interaction between the agencies began when Jones’ team began to develop the CONFIG product. Funding was provided for a few developers and a User Design Team (UDT) was formed consisting of about a dozen representatives from Sales. During the development aspect, we see the structural conditioning impacting the design of the project. At the same time a product to provide automated price quotes to the sales force (Price Quotation System - PQS) was under development. However, due to the stovepipe organizational philosophy, the two development groups never interacted. And while the members of the UDT began to argue for integration with PQS, the developers indicated that this would be too technically challenging and denied this request. After that occurred, the composition of the UDT began to change and become less representative. The less technical sales representatives dropped out leaving only the more experienced and more technical representatives involved. Eventually, a non-interactive, one-way linkage was implemented. CONFIG was faced with rejection by the sales force for the following reasons, the flaw design concept described above which made using it difficult and time consuming and the sales incentives which did not compensate the sales people for correct configurations.

What we see here is that Jones, following his situational logic, introduced a competitive contradiction to the existing structures by introducing the CONFIG system as a modification to the sales process. However, due to the stovepipe organization it represented no threat to the sales organization. The sales organization thus was never motivated to organize to oppose the implementation of the system and thus remained a primary agency. It simply stopped paying attention to the CONFIG system.

\section{Structural Elaboration}

The reasons for the failure of acceptance of the CONFIG system by the Sales organization can be seen in the power analysis of the elaboration phase. In the interactions between the agents, the resource alignments did not change. Jones' team while producing a technically successful product simply did not have any bargaining power. The system they produced was not something that the salespeople believed that they needed. While the sales force recognized the sanction of improving configurations, the system produced was simply too difficult to use and slowed down their sales cycle. Additionally, while Jones' team had the sanction of being recognized as having saved the company millions of dollars, the stovepipe 
organizational structure prevented this from being a bargaining chip Similarly, the sales compensation system provided a strong incentive to not bother with correcting the configurations. Left without bargaining power, Jones' team had no negotiating strength, which left them no ability to bargain for or force utilization of the system. The sales force therefore chose not to utilize the system. Jones' attempted competitive contradiction logic failed.

\section{What Should Jones Have Done to Be Successful?}

We saw that this development effort failed because a poor design concept rendered the system too difficult to use by the sales force for the usefulness it provided and because Jones' team lacked the negotiating strength to force use of the system. The flawed design concept arose because the stovepipe organizational structure prevented joint development of CONFIG with the PQS system.

Using the MAAD theory, we see that Jones could have taken a number of steps. He could have enlisted the support of upper management to create competitive contradictions in terms of the stovepipe organizational structure, which would have created an environment in which his development team could have worked with the PQS development team. Alternatively, Jones could have emphasized the contradiction between ideological propositions 1 and 6. By adopting an elimination strategy focused on creating an ideology of no configuration errors, he could have removed the sanction of sales force configuration errors, which would have given him more negotiating strength to persuade the sales force to use the system.

\section{Why the CONFIG Project was Allowed to Escalate Commitment}

Space constrains us from doing a full morphogenetic analysis, but a brief discussion may illuminate why the project escalated. The structure under consideration here is the CONFIG project itself. There were agencies that were advocating the termination of the project. Jones and Smith were advocating its continuance. Why did management allow the project to continue? Ideological structure 8 created an environment in which the company believed that a project in which the advocate could find funding should be allowed to continue. This structure created sanction for the project. Jones and his team found this as a strong contribution to its bargaining power and hence its negotiating strength. Additionally, Jones' credibility continued unabated also contributing to his negotiating strength. Thus the advocates of termination lacked ability to achieve their goal of discontinuing CONFIG.

\section{Why The CONFIG Project was Terminated}

Subsequent to those events, Keil (1995) reports that two events occurred. First, it is reported that a morphogenetic sequence occurred (insufficient detail is provided to analyze this cycle) in which ideological structure 8 was destroyed. The company entered a period of economic difficulty and there was pressure on management that resulted in belt tightening and the culture that propagated projects such as CONFIG was destroyed removing a source of sanction. Second, Jones died which resulted in an additional loss of sanction for the project. Without these sources of sanction, the bargaining power and hence negotiating strength of the project was greatly reduced leading to the success of those agencies advocating its termination.

\section{DISCUSSION AND LIMITATIONS}

The MAAD approach provides some interesting additions to the work of Keil. Keil described 13 factors that promoted escalation within the organization and five events that caused de-escalation of the project. His approach was one of variance analysis. He does not however provide the engine that allows us to show why those factors caused escalation or why those events promoted de-escalation. The MAAD approach, which is more process oriented, provides an explanatory engine that allows us to evaluate those factors and to describe how structural conditions changed to allow de-escalation. The use of the morphogenetic analytical approach allows us to qualitatively evaluate the contributions of each factor to conditioning the actions of the agents and also allows us to describe through the power analysis how those factors impacted the elaboration of the resultant structures. Through the application of the morphogenetic cycle to four situations (three explicitly described, one by implication), we were able to examine why CONFIG was not adopted in the sales organization, why it escalated, how the conditions changed to allow it to de-escalate and why it ultimately de-escalated.

Second, this approach allows us to disentangle psychological from sociological factors. Keil lists a number of factors that are psychological such as high degree of personal responsibility, errors in information processing, and an emotional attachment to the project. These factors, while important to motivating Jones and his team, are not factors that describe why the organization continued supporting the project. Thus in the variance approach different levels of analysis are mixed. In the MAAD approach, these psychological factors are not considered in the power analysis of the elaboration of structures. 
Third, this approach adds to that of Keil, by adding the effects of context into the analysis. It provides a mechanism by which to analyze social structures and their effects on the agencies involved and to explain how this context affects their behavior. By means of the analysis of the necessary/contingent nature of the structure and the complementary/contradictory nature of the relationships within it, we can see the directional guidance it provides to the agencies. By the analysis of the bargaining power engendered by the allocation of resources and the ensuing negotiating strength derived from it, we can explain how the contextual factors influenced the subsequent development of the social structures.

As a limitation, this approach does not deal with the psychological factors that drive why the agencies choose the projects that they do. The MAAD analysis does indicate that structures provide direction guidance by indicating that as the projects that they choose succeed or fail it directs them to chose whether to desire sustain, eliminate or change the structures.

A second limitation is that this is a secondary case study, which relies on the data that is presented in the case at hand. As such it is only an exemplar of the methodology. This should be applied to more cases and actual data to prove the methodology in practice.

This methodology is applicable to areas discussing social level change, e.g. change in business process such as system implementations, outsourcing arrangements, and development activities at the social level. It is not applicable for analysis of purely psychological phenomena such as attitudes

\section{CONCLUSIONS}

This secondary study has shown that using Archer's MAAD social theory allows explanatory purchase for identifying reasons for project success, escalation and de-escalation of a project and identified ways to improve the performance of the development effort. It has shown that social structures, both ideological and material, should be considered in the development of explanations for phenomena. In particular, it places focus on the reasons for why structural change occurred or did not occur and therefore is useful for examining cases where the introduction or use of information systems caused changes in social structures such as business processes, organizations or culture.

\section{REFERENCES}

Archer, M. (1998). Realism and Morphogenesis. Critical Realism Essential Readings. M. Archer, R. Bhaskar, A. Collier, T. Lawson and A. Norrie. London, Routlege.

Archer, M. S. (1988). Culture and Agency, The Place of Culture in Social Theory. Cambridge, Cambridge University Press.

Archer, M. S. (1995). Realist Social Theory: the morphogenetic approach. Cambridge, Cambridge University Press.

Jones, M. and H. Karsten (2008). "Gidden's Structuration Theory and Information systems Research." MIS Quarterly 32(1): 127-157.

Jones, M., W. J. Orlikowski, et al. (2004). Structuration Theory and Information Systems: A Critical Reappraisal. Social Theory and Philosophy for Information Systems. J. Mingers and L. Willcocks. Chichester, John Wiley \& Sons: 297328.

Keil, M. (1995). "Pulling the Plug: Software Project Management and the Problem of Project Escalation." MIS Quarterly 19(4): 421-477.

Orlikowski, W. J. (2000). "Using Technology and Constituting Structures: A Practice Lens for Studying Technology in Organizations." Organization Science 11(4): 404-428.

Orlikowski, W. J. (2005). "Material Works: Exploring the Situated Entanglement of Technological Performativity and Human Agency." Scandinavian Journal of Information Systems 17(1): 183-186.

Rose, J., M. Jones, et al. (2005). "Socio-Theoretic Accounts of IS: The Problem of Agency." Scandinavian Journal of Information Systems 17(1): 133-152.

Rubenstein, D. (2007) "Standish Group Report: There's Less Development Chaos Today." Software Development Times on the Web.

Wikipedia. (2009, 10/31/2009). "Social Theory." Wikipedia, from http://en.wikipedia.org/wiki/Social_theory. 